\title{
Altura de voo de bostriquídeos (Coleoptera: Bostrichidae) coletados em Floresta Tropical Semidecídua, Mato Grosso
}

\author{
Otávio Peres Filho ${ }^{1}$, Janaína Iris Barbosa ${ }^{1}$, Marcelo Dias de Souza ${ }^{1}$, Alberto Dorval ${ }^{1}$ \\ ${ }^{1}$ Universidade Federal de Mato Grosso, Departamento de Engenharia Florestal, Av. Fernando Corrêa da Costa, 2367, CEP 78125-070, Cuiabá, MT, Brasil
}

"Autor correspondente:

peres@ufmt.br

Termos para indexação:

Coleobrocas

Besouros pulverizadores

Amazônia legal

Altura de voo

Index terms:

Woodborer

Powder beetles

Amazon

Flight height

\section{Histórico do artigo:}

Recebido em 25 jun 2011

Aprovado em 05 mar 2012

Publicado em 30 mar 2012

doi: 10.4336/2012.pfb.32.69.101
Resumo - O objetivo deste trabalho foi determinar a altura de voo das espécies da família Bostrichidae, bem como determinar os índices faunísticos das espécies coletadas em Floresta Estacional Semidecídua, no período de abril de 2000 a março de 2001, na fazenda Maracaí, localizada no município de Sinop, Mato Grosso. Foram testadas sete alturas com 21 armadilhas etanólicas, sendo distribuídas três armadilhas por altura. $\mathrm{O}$ atrativo utilizado foi o álcool comercial. Inicialmente as coletas foram realizadas quinzenalmente, e depois, mensalmente. O material coletado foi identificado por comparação com exemplares da coleção do Laboratório de Proteção Florestal (LAPROFLOR) da Universidade Federal de Mato Grosso. Foram coletados 141 bostriquídeos, divididos em cinco espécies: Bostrychopsis uncinata, Micrapate brasiliensis, Micrapate germaini, Xyloperthella piceae e Xyloprista praemorsa. A coleta da maioria das espécies de Bostrichidae foi significativamente maior na altura de $30 \mathrm{~m}$. Dentre as espécies capturadas, $X$. picea foi a mais frequente e dominante. Essa espécie apresentou picos superiores de ocorrência nos meses de junho e fevereiro, e mostrou-se significativamente diferente das demais espécies quanto à ocorrência. No período de estudo não ocorreu correlação significativa entre os fatores climáticos estudados e ocorrência das espécies.

\section{Flight height of bostrichids (Coleoptera: Bostrichidae) collected in Tropical Semideciduous Forest, Mato Grosso, Brazil}

\begin{abstract}
The objective of this study was to determine the flight height of species of Bostrichidae family, as well as determine the faunistic index of species collected in Tropical Semideciduous Forest, in the period to April of 2000 at March of 2001, in Maracaí farm, located in the municipality of Sinop, Mato Grosso, Brazil. Seven heights with 21 ethanolic traps were tested, with three traps by height. The bait used was the commercial alcohol and the collects were proceeded every fifteen days, and latter on monthly. The material collected was identified by comparison with specimens of the Forest Protection laboratory collection, from the Federal University of Mato Grosso. Were collected 141 individuals from five species: Bostrychopsis uncinata, Micrapate brasiliensis, Micrapate germaini, Xyloperthella piceae and Xyloprista praemorsa. The collects of most species of Bostrichidae, were significantly higher at 30 meters. The most frequent and dominant species captured was $X$. picea. This species presented the higher peak of occurrence in June and February, significantly different from the other species. In the studying period, it was not observed significant correlation between climate and the occurrence of the studied species.
\end{abstract}




\section{Introdução}

Os coleópteros são dominantes nos trópicos, possuindo diversas espécies pragas de grande importância em razão dos seus danos na área florestal, equivalendo-se em importância aos isópteros, os quais são conhecidos como as pragas mais destrutivas da madeira (Gray, 1972). Muitos trabalhos demonstram a importância de coleobrocas no Brasil, que causam danos tanto a árvores em pé, quanto a madeira estocada em serrarias (Zanuncio et al., 2005; Flechtmann \& Gaspareto, 1997; Abreu et al., 2002).

O controle das coleobrocas é de extrema dificuldade, uma vez que o controle químico é feito tarde demais e a aplicação localizada dos produtos químicos nem sempre atinge a população em trânsito (Vité, 1971). Além disso, o controle direto não leva em conta o controle natural (parasitos, predadores e competidores) (Berti Filho, 1979).

A família Bostrichidae compõe um grupo bem delimitado dentro da ordem Coleoptera, sendo composto por espécies mais adaptadas ao regime xilófago (Lesne, 1924). De maneira geral, estes insetos, tanto no estágio larval quanto no estágio adulto, se nutrem efetivamente dos tecidos lenhosos dos vegetais (Fletchmann et al., 1996; Peters et al., 2002). Na família Bostrichidae muitas espécies são conhecidas como pragas de grãos e sementes e de broca de madeira armazenada (Pereira et al., 1997; Chander \& Bhargava, 2006). Estas espécies são conhecidas como "besouros pulverizadores da madeira", ou seja, ao abrirem galerias, transformam a madeira em pó, depreciando o valor de produtos, como peças estruturais e lâminas (Peres Filho et al., 2006).

Os bostriquídeos possuem numerosos representantes na região tropical, onde provocam prejuízos importantes, como a espécie Dinoderus minutus Fabricius, que tem ocorrido com proliferação acentuada em lâminas de espécies tropicais estocadas (Matoski \& Rocha, 2006). A maioria não tem hospedeiros específicos, podendo atacar grupos botânicos, por vezes bastante diferentes, manifestando assim uma elevada polifagia. Os adultos frequentemente apresentam um regime alimentar diferente das larvas, alimentando-se muitas vezes de ramos e troncos de árvores vivas plenas de vigor, enquanto as larvas se alimentam de tecido lenhoso de árvores recentemente mortas ou abatidas (Carvalho, 1971; Puebla et al., 2007).

Atualmente, os bostriquídeos não se constituem um problema de ordem econômica em reflorestamentos no Brasil, possivelmente a principal razão para que as informações sobre as coleobrocas desta família sejam escassas. A gradual diminuição das áreas de vegetação nativa, local preferencial de desenvolvimento dos Bostrichidae, talvez contribua para a adaptação dessas coleobrocas às espécies florestais exóticas, pertencentes aos gêneros Pinus ou Eucalyptus, podendo então se constituir em pragas (Flechtmann et al., 1997).

O estudo da altura de voo de espécies de insetos com potencial para praga possibilita avaliar até que ponto podem ocorrer os ataques, além de propiciar informações para um melhor entendimento das áreas atacadas, bem como fornecer informações que poderão ser utilizadas em estratégias de controle, revelando espécies já descritas como pragas em florestas plantadas. Portanto, o estudo teve como objetivo conhecer a altura de voo dos bostriquídeos pela coleta das espécies em diferentes alturas, além de determinar as flutuações populacionais e estabelecer estudos de correlações significativas entre as condições meteorológicas e a ocorrência das espécies.

\section{Material e Métodos}

Os levantamentos foram realizados na fazenda Maracaí, pertencente à Maracaí Florestal e Indústria Ltda., que está situada no município de Sinop, à cerca de $60 \mathrm{~km}$ do centro urbano, no estado de Mato Grosso. Esta região está localizada nas coordenadas $11^{\circ} 24,45^{\prime}$ S; 55¹9,30' W e 423 m de altitude em relação ao nível do mar. A formação básica da vegetação natural da região, em sua maioria, é do tipo Floresta Estacional Semidecidual. A área estudada não sofreu modificações no seu estado natural por ações antrópicas.

As amostras foram coletadas no período de abril de 2000 a março de 2001. Para a coleta dos insetos foram utilizadas 21 armadilhas etanólicas, modelo escolitídeoCuritiba (Marques, 1984), contendo álcool comercial 98\% GL e álcool 70\%, no porta isca e no recipiente de coleta, respectivamente. As armadilhas foram instaladas em diferentes alturas: $1,5,11,15,21,30$ e 35 metros, em relação ao solo, em uma torre metálica situada no interior da floresta amazônica, utilizada para estudos meteorológicos, com $40 \mathrm{~m}$ de altura total. Para cada altura foram instaladas três armadilhas nas extremidades da torre equidistantes, a $2 \mathrm{~m}$ de distância.

As coletas foram realizadas quinzenalmente, e depois, mensalmente, para efeito de análise. As armadilhas permaneceram no campo durante todo o tempo de 
realização do estudo. A cada duas semanas os insetos eram coletados e o álcool do porta isca e do recipiente de coleta eram repostos. Os insetos coletados foram transportados em garrafas plásticas contendo álcool $70 \%$, devidamente etiquetadas, individualizadas por armadilha e trazidas para o Laboratório de Proteção Florestal (LAPROFLOR) da Universidade Federal de Mato Grosso (UFMT). Os insetos foram triados e secos em estufa a $60^{\circ} \mathrm{C}$ por 72 horas e, posteriormente, foram efetuadas a contagem e identificação das espécies.

A identificação taxonômica dos indivíduos foi realizada mediante comparações diretas com exemplares existentes na coleção entomológica do LAPROFLOR, onde os insetos foram identificados em nível de espécie. A análise quantitativa foi realizada através da contagem direta dos exemplares identificados. Em seguida, os dados foram submetidos a estudos faunísticos, de acordo com o uso do software ANAFAU (Moraes et al., 2003) e foram calculadas a dominância, a frequência e a constância das espécies.

A diversidade foi calculada pela equação de Margalef (1951), que representa a razão entre o número de espécies no ambiente menos um e o logaritmo neperiano (Ln) do número de indivíduos do ambiente estudado. Foram determinadas as flutuações populacionais de todas as espécies coletadas, sendo estabelecida a correlação dos fatores climáticos com a ocorrência dos insetos coletados, utilizando-se os dados do período da coleta, como temperatura média mensal, umidade relativa do ar mensal e precipitação pluvial total mensal.

Para verificar as possíveis diferenças significativas que podem ocorrer em relação às diferentes alturas de voo dos insetos, os dados foram analisados estatisticamente em esquema fatorial (espécies $\mathrm{x}$ alturas) com três repetições (armadilhas). Os dados foram submetidos à análise de variância (ANOVA) e as médias foram comparadas entre si pelo teste de Tukey, ao nível de $5 \%$ de probabilidade. As análises foram executadas através do software Assistat, versão 7.6 beta (Silva \& Azevedo, 2002).

Nas analises estatísticas, levando-se em conta a distribuição dos valores das coletas para as espécies de Bostrichidae nas diferentes alturas, utilizouse a transformação dos dados usando a fórmula $\sqrt{X+0,5}$ para a uniformização destes e normalidade dos dados (Phillips, 1990; Flechtmann et al., 1996; Flechtmann et al., 1997; Dorval et al., 2004).

\section{Resultados e discussão}

Nas 21 armadilhas etanólicas distribuídas em sete alturas, foram coletados 141 indivíduos, distribuídos em quatro gêneros e cinco espécies: Bostrichopsis uncinata (German, 1824), Micrapate brasiliensis (German, 1824), Micrapate germaini (German, 1824), Xyloperthella picea (Oliver, 1790) e Xyloprista praemorsa (Erichson, 1847). A espécie $X$. picea foi a mais representativa, apresentando um total de $73,8 \%$ das coletas dos espécimes, enquanto a espécie $M$. brasiliensis foi a que teve menor número de indivíduos coletados, ocorrendo apenas uma vez ao longo de um ano. No estudo de Flechtmann et al. (1997) ocorreram seis espécies distribuídas em seis gêneros, sendo a mais representativa a espécie $B$. uncinata. As armadilhas instaladas a $30 \mathrm{~m}$ em relação ao solo foram as que obtiveram os maiores números de indivíduos (Tabela 1).

$\mathrm{O}$ índice de diversidade foi baixo $(0,81)$. Segundo Margalef (1972), esse índice raramente ultrapassa o valor

Tabela 1. Espécies e quantidade de indivíduos coletados em armadilhas etanólicas instaladas em diferentes alturas. Fazenda Maracaí, Sinop-MT, 2000-2001.

\begin{tabular}{|c|c|c|c|c|c|c|c|c|c|}
\hline \multirow{2}{*}{ Espécie } & \multicolumn{7}{|c|}{ Altura de Armadilhas (m) } & \multirow{2}{*}{ Total } & \multirow{2}{*}{$\%$} \\
\hline & 1 & 5 & 11 & 15 & 21 & 30 & 35 & & \\
\hline Bostrichopsis uncinata & - & - & 2 & 1 & 4 & 4 & 3 & 14 & 9,9 \\
\hline Micrapate brasiliensis & - & - & - & - & - & 1 & - & 1 & 0,7 \\
\hline Micrapate germaini & - & - & 1 & 1 & 1 & 1 & 4 & 8 & 5,7 \\
\hline Xyloperthella picea & - & - & 4 & 6 & 7 & 53 & 34 & 104 & 73,8 \\
\hline Xyloprista praemorsa & - & - & - & - & 1 & 8 & 5 & 14 & 9,9 \\
\hline Total de espécies & 0 & 0 & 3 & 3 & 4 & 5 & 4 & 5 & - \\
\hline Total de indivíduos & 0 & 0 & 7 & 8 & 13 & 67 & 46 & 141 & 100 \\
\hline Diversidade & \multicolumn{9}{|c|}{0,81} \\
\hline
\end{tabular}


de 4,5,variando normalmente entre 1,5 e 3,5. Os valores baixos representam um resultado da maior dominância de alguns grupos taxonômicos em detrimento da maioria.

Considerando-se o total de espécies coletadas nas sete alturas deste estudo, de acordo com o método de Sakagami \& Larroca (1967), ocorreram um total de quatro espécies não dominantes e uma espécie dominante. A dominância expressa a influência sofrida por uma determinada espécie, considerada pouco abundante e que pode desempenhar função mais importante dentro de uma comunidade, por outra espécie, considerada mais abundante (Silveira Neto et al., 1976). Quanto à frequência, verificaram-se quatro espécies frequentes e uma espécie muito frequente e, de acordo com a constância, verificou-se duas espécies acessórias, uma espécie acidental e duas espécies constantes (Tabela 2).

Flechtmann et al. (1996) e Flechtmann et al. (1997) estudaram a altura de voo de alguns bostríquideos em ambientes com reflorestamentos de Pinus spp. , na qual a espécie mais frequente foi $B$. uncinata em ambos os estudos. Segundo Alcioli \& Ximenes (1975), a espécie $B$. uncinata já foi relatada atacando reflorestamento de eucalipto no interior do Ceará. Nesse estudo, em condição de floresta natural, $B$. uncinata não se mostrou dominante, apesar de frequente. $X$. picea foi a que teve maior ocorrência, sendo dominante em relação às demais espécies, porém, vale ressaltar que os resultados distintos são devidos aos diferentes ambientes de estudo.

Segundo Clemente (1995), a resistência do meio sobre a proliferação das espécies se reflete na dominância, constância e abundância. Uma alta porcentagem de indivíduos acidentais indica uma resistência à proliferação das espécies, enquanto as classificações consideradas abundantes, constantes e dominantes

Tabela 2. Relação das espécies, quantidade de indivíduos e índices faunísticos de Bostrichidae coletados em armadilhas etanólicas em diferentes alturas. fazenda Maracaí, Sinop, MT, 2000-2001.

\begin{tabular}{lcccc}
\hline \multirow{2}{*}{ Espécies } & Quantidadede & \multicolumn{3}{c}{ Índices faunísticos } \\
\cline { 3 - 5 } & Indivíduos & Dominância & Frequência & Constância \\
\hline Bostrichopsis uncinata & 14 & ND & $\mathrm{F}$ & $\mathrm{Y}$ \\
Micrapate brasiliensis & 1 & $\mathrm{ND}$ & $\mathrm{F}$ & $\mathrm{Z}$ \\
Micrapate germaini & 8 & $\mathrm{ND}$ & $\mathrm{F}$ & $\mathrm{Y}$ \\
Xyloperthella picea & 104 & $\mathrm{D}$ & $\mathrm{MF}$ & $\mathrm{W}$ \\
Xyloprista praemorsa & 14 & $\mathrm{ND}$ & $\mathrm{F}$ & $\mathrm{W}$ \\
\hline
\end{tabular}

Dominante (D); Não dominante (ND); Muito frequente (MF); Frequente (F); Constância (C); Constante (W); Acessória (Y) e Acidental (Z)

indicam o estabelecimento destas espécies dentro das comunidades.

Considerando todos os bostriquídeos coletados, podese notar que ocorreram picos populacionais distintos, observados nos meses de junho, agosto e fevereiro. A espécie $X$. picea apresentou maiores picos nos meses de junho e fevereiro (Figura 1A), diferindo dos resultados observados por Dall'Oglio \& Peres Filho (1997). Esses autores verificaram que em plantios de Hevea brasiliensis, no município de Itiquira, MT, a espécie apresentou picos populacionais de julho a novembro.

A espécie $X$. praemorsa teve maior pico no mês de dezembro, sendo estável no período de outubro a março (Figura 1B). A espécie B. uncinata teve maior ocorrência no mês de julho a janeiro (Figura 1C). Rocha et al. (2011) observaram maior quantidade de indivíduos dessa espécie em floresta de eucalipto nos meses de maio a outubro, período de estiagem na região. A espécie $M$. germaini teve ocorrência em julho a outubro e, posteriormente, voltou a ter ocorrência em dezembro e fevereiro (Figura 1D). A espécie $M$. brasiliensis apresentou apenas um espécime coletado no mês de outubro

Segundo Saunders \& Knoke (1967), as mudanças nos fatores climáticos influem direta ou indiretamente na flutuação populacional de insetos. Sendo assim, foi necessário conhecer esses fatores para uma melhor interpretação da comunidade de Bostrichidae. Foram verificadas as correlações entre as variáveis climáticas, temperatura, precipitação pluvial e umidade relativa na ocorrência das espécies, mas não foram consideradas as correlações com coeficientes inferiores a 0,60 , por 

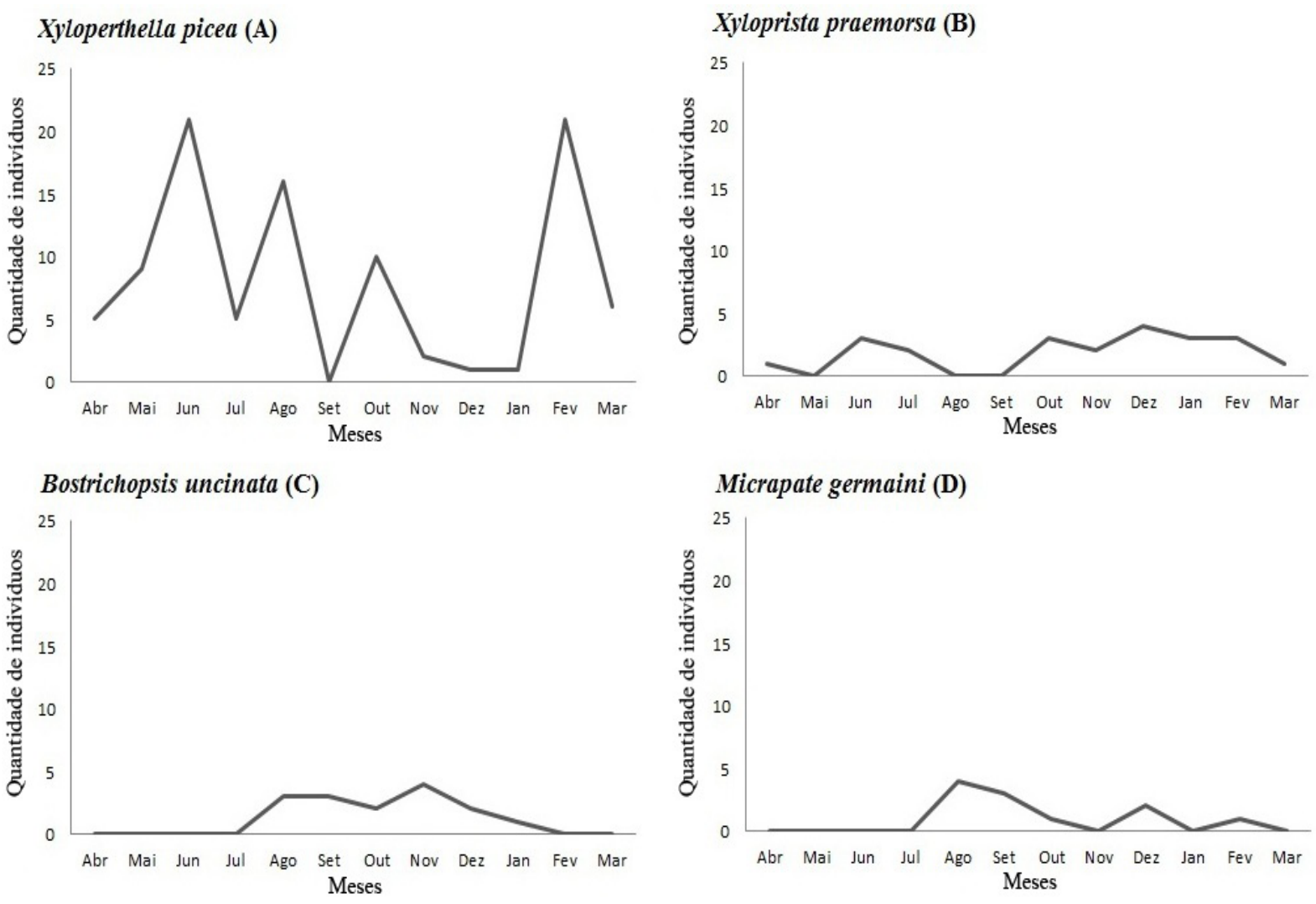

Figura 1. Flutuação populacional das espécies de Bostrichidae coletados em armadilhas etanólicas, em diferentes alturas. fazenda Maracaí, Sinop, MT, 2000-2001.

não explicarem satisfatoriamente o comportamento das espécies de insetos em relação aos fatores ambientais (Dorval, 2002). Foi constatado que não houve correlação entre as variáveis meteorológicas e a ocorrência das espécies, pois todas as correlações foram menores que 0,60. Rocha et al. (2011) relataram maior ocorrência de $X$. Picea em florestas de Eucalyptus camaldulensis Dehn. no período chuvoso. Entretanto, no estudo desenvolvido por esses autores houve maior quantidade de indivíduos da espécie $B$. laminifer, com ocorrência significativamente maior que as demais espécies de bostriquídeos coletados.

As espécies B. uncinata e X. praemorsa apresentaram uma quantidade significativamente maior de indivíduos na altura de $30 \mathrm{~m}$, porém, não houve diferença significativa com a altura de $35 \mathrm{~m}$. A espécie $M$. germaini apresentou maior quantidade de indivíduos na altura de $35 \mathrm{~m}$. A espécie $X$. picea apresentou maior quantidade de indivíduos na altura de $30 \mathrm{~m}$; quanto a sua variação ao longo do experimento, essa espécie sempre apresentou valores de indivíduos coletados mais expressivos em relação as demais espécies. O indivíduo de $M$. brasiliensis foi coletado na armadilha a $30 \mathrm{~m}$ (Tabela 4). Nas alturas de $1 \mathrm{~m}$ e $5 \mathrm{~m}$ não houve ocorrência de nenhuma espécie de bostriquídeo, não sendo, portanto, relacionadas na tabela 3 .

Analisando-se as médias de cada espécie nas diferentes alturas, verificou-se diferença significativa nas alturas de $30 \mathrm{~m}$ e $35 \mathrm{~m}$, o que corrobora com Flechtmann et al. (1997), que afirmaram que os bostriquídeos poderiam voar em alturas superiores a $10 \mathrm{~m}$ em relação ao solo. $\mathrm{Na}$ análise da quantidade total de indivíduos, verificou-se que $X$. picea apresentou maior ocorrência significativa em relação às demais espécies, principalmente nas alturas $30 \mathrm{~m}$ e $35 \mathrm{~m}$, sendo essas alturas as mais representativas numericamente para ambas as espécies.

A espécie $X$. picea foi a mais predominante nas coletas, sendo a altura de $30 \mathrm{~m}$ a preferencial de voo. Essa espécie já foi registrada por Andrade (1928) causando danos em Eucalyptus spp., sendo também relatada por Berti Filho (1981) atacando árvores matrizes de Eucalyptus spp. com 15 anos de idade no estado de 
Tabela 3. Quantidade média dos indivíduos da família Bostrichidae, coletados em armadilhas etanólicas em diferentes alturas. Fazenda Maracaí, Sinop-MT, 2000-2001.

\begin{tabular}{|c|c|c|c|c|c|}
\hline \multirow{2}{*}{ Espécies } & \multicolumn{5}{|c|}{ Alturas (m) ${ }^{1}$} \\
\hline & 11 & 15 & 21 & 30 & 35 \\
\hline Bostrichopsis uncinata & $1,05 \mathrm{abAB}$ & $0,87 \mathrm{bAB}$ & $1,34 \mathrm{aA}$ & $1,34 \mathrm{bA}$ & $1,22 \mathrm{bA}$ \\
\hline Micrapate brasiliensis & $0,71 \mathrm{bA}$ & $0,71 \mathrm{bA}$ & $0,71 \mathrm{bA}$ & $0,87 \mathrm{cA}$ & $0,71 \mathrm{cA}$ \\
\hline Micrapate germaini & $0,87 \mathrm{bAB}$ & $0,87 \mathrm{bAB}$ & $0,87 \mathrm{bAB}$ & $0,87 \mathrm{cAB}$ & $1,34 \mathrm{bA}$ \\
\hline Xyloperthella picea & $1,34 \mathrm{aC}$ & $1,55 \mathrm{aC}$ & $1,67 \mathrm{aC}$ & $4,25 \mathrm{aA}$ & $3,42 \mathrm{aB}$ \\
\hline Xyloprista praemorsa & $0,70 \mathrm{bB}$ & $0,70 \mathrm{bB}$ & $0,87 \mathrm{bB}$ & $1,77 \mathrm{bA}$ & $1,46 \mathrm{bA}$ \\
\hline $\mathrm{F}$ & \multicolumn{5}{|c|}{$24,34^{*}$} \\
\hline $\mathrm{CV}(\%)$ & \multicolumn{5}{|c|}{17,22} \\
\hline
\end{tabular}

São Paulo, broqueando troncos de árvores com $45 \mathrm{~cm}$ de diâmetro e causando vários danos, devido ao fato de suas galerias serem no sentido horizontal.

\section{Conclusões}

Pode-se concluir que a altura influencia na quantidade de indivíduos e espécies, sendo que os bostriquídeos têm maior ocorrência em alturas mais elevadas. As variáveis climáticas testadas no período de estudo não influenciaram na ocorrência dos indivíduos.

\section{Referências}

ABREU, R. L. S.; SALES-CAMPOS, C.; HANADA, R. E.; VASCONCELLOS, F. J.; FREITAS, J. A. Avaliação de danos por insetos em toras estocadas em indústrias madeireiras de Manaus, Amazonas, Brasil. Revista Árvore, Viçosa, MG, v. 26, p. 789-796, 2002.

ALCIOLI, A.; XIMENES, A. P. Bostrichopsis uncinata (Germar, 1824), praga de Eucalyptus sp. no Estado do Ceará, Brasil. Fitossanidade. Fortaleza, v. 1, n. 3, p. 98-99, 1975.

ANDRADE, E. N. Contribuição para o estudo da entomologia florestal paulista. Boletim agrícola, São Paulo, v. 29, n. 8, p. 446453, 1928.

BERTI FILHO E. Insetos associados a plantações de espécies do gênero Eucalyptus nos estados da Bahia, Espirito Santo, Mato Grosso do Sul, Minas Gerais e São Paulo. 1981. 176 f. Tese (Doutorado em Entomologia) Escola Superior de Agricultura "Luiz de Queiroz”, Universidade de São Paulo, Piracicaba, SP.

BERTI FILHO, E. Coleópteros de importância florestal: 1- Scolytidae. IPEF, Piracicaba, SP, v. 19, p. 39-43, 1979.

CARVALHO, J. P. Introdução à entomologia florestal de Angola. Angola: Nova Lisboa, 1971. 314 p.
CHANDER, R.; BHARGAVA, M. C. Development of lesser grain borer, Rhizopertha dominica (FAB.) on different hosts. Journal of Insect Science, Ludhiana, v. 19, n. 1, p. 13-15, 2006.

CLEMENTE, A. T. C. Análise de populações de Lepidoptera em comunidades florestais de Araucariaangustifolia, Eucalyptusgrandis e Pinus taeda. 1995, 89 f. Dissertação (Mestrado em Engenharia Florestal) - Universidade Federal do Paraná, Curitiba.

DALL'OGLIO, O. T.; PERES FILHO. O. Levantamento e flutuação de populacional de coleobrocas em plantios homogêneos de seringueira em Itiquira-MT. Scientia Forestalis, Piracicaba, v. 51, n. 2, p. 49-58, 1997.

DORVAL, A. Levantamento populacional de coleópteros com armadilhas etanólicas em plantios de Eucalyptus e em uma área com vegetação de cerrado no município de Cuiabá-MT. 2002. 143 f. Tese (Doutorado em Ciências Biológicas) - Universidade Federal do Paraná, Curitiba.

DORVAL, A.; PERES FILHO, O.; MARQUES, E. N. Levantamento de Scolytidae (coleoptera) em plantações de Eucalyptus spp. em Cuiabá, estado de Mato Grosso. Ciência Florestal, Santa Maria, v. 14, n. 1, p. 47-58, 2004.

FLECHTMANN, C. A. H.; GASPARETO, C. L.; TEIXEIRA, E. Altura de voo de Bostrichidae (Coleoptera) em Pinus caribaea $v$. hondurensis em Agudos, SP. Revista Instituto Florestal, São Paulo, v. 9, n. 1, p. 19-26, 1997.

FLECHTMANN, C. A. H; TEIXEIRA E. P; GASPARETO, C. L. Bostrichidae (Coleoptera) coletados em armadilhas iscadas com etanol em pinheiros de Agudos, SP. Revista Instituto Florestal, São Paulo, v. 1, p. 17-44, 1996.

FLETCHMANN, C. A. H.; GASPARETO, C. L. Scolytidae em pátio de serraria da fábrica Paula Souza (Botucatu/SP) e fazenda Rio Claro (Lencóis Paulista/SP). Scientia Forestalis, Piracicaba, n. 51, p. 61-75, 1997.

GRAY, B. Economic tropical forest entomology. Annual Review Entomology, v. 17, p. 313-354, 1972. 
LESNE, P. Les Coléoptères Bostrychides de l'Afrique tropicale française. Paris: Presses Universitaires de France: P. Lechevalier, 1924. $288 \mathrm{p}$.

MARGALEF, R. Diversidad de espécies em las comunidades naturales. Publicaciones del Institutode Biologia Aplicada e Barcelona, Barcelona, v. 6, p. 59-72, 1951.

MARGALEF, R. Homage to Evelyn Hutchinson, or why is there an upper limit to diversity. Transactions of the Connecticut Academy of Arts and Sciences, New Haven, v. 44, p. 211-235, 1972.

MARQUES, E. N. Scolytidae e Platypodidae em Pinus taeda. 1984. 65 f. Dissertação (Mestrado em Engenharia Florestal) - Universidade Federal do Paraná, Curitiba.

MATOSKI, S. L. S.; ROCHA, M. P. Influência do fotoperíodo no ataque de Dinoderus minutus Fabricius (Coleoptera: Bostrichidae) em lâminas torneadas de espécies tropicais. Revista floresta, Curitiba, v. 36, n. 3, 2006.

MORAES, R. C. B.; HADDAD, M. L.; SILVEIRA NETO, S.; REYES, A. E. L. Software para análise faunística. In: SIMPÓSIO DE CONTROLE BIOLÓGICO, 8., 2003, São Pedro, SP. Resumos. Piracicaba, SP: SEB, 2003. p. 195

PEREIRA, P. R. V. S.; FURIATTI, R. S.; LAZZARI, F. A.; PINTO JUNIOR, A. R. Avaliação de Inseticidas no Controle de Sitophilus oryzae (L.) (Coleoptera: Curculionidae) e Rhyzopertha dominica (Fab.) (Coleoptera: Bostrichidae) em Milho Armazenado. Anais da Sociedade Entomológica do Brasil, Londrina, v. 26, n. 3, p. 411-416, 1997.

PERES FILHO, O.; DORVAL, A.; BERTI FILHO, E. A entomofauna associada à Teca, Tectona grandis L.f. no Estado de Mato Grosso. Piracicaba, SP: IPEF; 2006. 58 p.

PETERS, B. C.; CREFFIELD, J. W.; ELDRIDGE, R. H. Lyctine (Coleoptera: Bostrichidae) pests of timber in Australia: a literature review and susceptibility testing protocol. Australian Forestry, Queen Victoria, v. 65, p. 107-119, 2002.
PHILLIPS, T. W. Responses of Hylastes salebrosus to turpentine, ethanol, and pheromones of Dendroctonus (Coleoptera: Scolytidae). The Florida Entomologist, Gainesville, v. 73, n. 2, p. 286-292, 1990.

PUEBLA, P.; LÓPEZ-COLÓN, J. I.; BAENA, M. Los Bostrichidae Latreille, 1802 de la fauna íbero-balear (Coleoptera). Heteropterus Revista de Entomología, v. 7, n. 2, p. 147-227, 2007.

ROCHA, J. R. M.; DORVAL, A.; PERES FILHO, O.; SOUZA, M. D.; COSTA, R. B. Análise da ocorrência de Coleópteros em plantios de Eucalyptus camaldulensis Dehn. em Cuiabá, MT. Floresta e Ambiente, Seropédica, v. 18, n. 4, p. 343-352, 2011.

SAKAGAMI, S. F.; LAROCA, S. Observations on the bionomics of some neotropical Xylocopini bees, with some comparative biofaunistic notes (Hymenoptera, Anthophoridae). Journal of the Faculty of Science, Hokkaido, v. 18, p. 57-127, 1967.

SAUDERS, J. L.; KNOKE, J. K. Diurnal emergence of Xyleborus ferrugineus (Coleoptera: Scolytidae) from cacao trunks in Ecuador and Costa Rica. Annals of the Entomological Society of America, Columbus, v. 60 n. 4, p. 1094-1096, 1967.

SILVA, F. A. S.; AZEVEDO, C. A. V. Versão do programa computacional Assistat para o sistema operacional Windows. Revista Brasileira de Produtos Agroindustriais, Campina Grande, v. 4, n. 1, p. 71-78, 2002.

SILVEIRA NETO, S.; NAKANO, O.; BARDIN, D.; VILLA NOVA, N. A. Manual de ecologia dos insetos. Piracicaba, SP: Ceres, 1976. $420 \mathrm{p}$.

VITÉ, J. P. Silviculture and the management of bark beetle pests. ln: CONFERENCE ON ECOLOGICAL ANIMAL CONTROL BY HABITAT MANAGEMENT, 3. Proceedings... Tallahassee: Tall Timbers Research Station, 1971. p. 155-68.

ZANUNCIO, J. C.; SOSSAI, M. F.; FLECHTMANN, C. A. H.; ZANUNCIO, T. V.; GUIMARÃES, E. M.; ESPINDULA, M. C. Plants of an Eucalyptus clone damaged by Scolytidae and Platypodidae (Coleoptera). Pesquisa Agropecuária Brasileira, DF, v. 40, p. 513-515, 2005. 
